\title{
Correction to: Human papillomavirus type 16 causes a defined subset of conjunctival in situ squamous cell carcinomas
}

\author{
Heather Griffin ${ }^{1} \cdot$ Hardeep Singh Mudhar ${ }^{2} \cdot$ Paul Rundle $^{3} \cdot$ Aslam Shiraz $^{1} \cdot$ Radma Mahmood $^{4} \cdot$ Nagayasu Egawa $^{1}$. \\ Wim Quint ${ }^{5} \cdot$ Ian G. Rennie ${ }^{3} \cdot$ John Doorbar $^{1}$
}

Published online: 15 October 2019

(c) United States \& Canadian Academy of Pathology 2019

\section{Correction to: Modern Pathology} https://doi.org/10.1038/s41379-019-0350-5

Author 'Hardeep Singh Mudhar' was listed in the original article as given name: Hardeep, family name: Singh
Mudhar. However, this should be listed as given name: Hardeep Singh, family name: Mudhar. This has been addressed by means of this correction article.

John Doorbar

jd121@cam.ac.uk

1 Department of Pathology, University of Cambridge, Tennis Court Road, Cambridge CB2 1QP, UK

2 National Specialist Ophthalmic Pathology Service, Department of Histopathology, E-Floor, Royal Hallamshire Hospital, Glossop Rd, Sheffield S10 2JF, UK

3 Sheffield Ocular Oncology Service, Department of Ophthalmology, Royal Hallamshire Hospital, Glossop Rd, Sheffield S10 2JF, UK

4 Francis Crick Institute, Mill Hill Laboratory, The Ridgeway, Mill Hill, London NW7 1AA, UK

5 Delft Diagnostic Laboratories, Visseringlaan 25, 2288 ER Rijswijk, The Netherlands 